\title{
Binding of Quasi-Two-Dimensional Biexcitons
}

Birkedal, Dan; Singh, Jai; Vadim, Lyssenko; Østergaard, John Erland; Hvam, Jørn Märcher

\section{Published in:}

Physical Review Letters

Link to article, DOI:

10.1103/PhysRevLett.76.672

Publication date:

1996

Document Version

Publisher's PDF, also known as Version of record

Link back to DTU Orbit

Citation (APA):

Birkedal, D., Singh, J., Vadim, L., Østergaard, J. E., \& Hvam, J. M. (1996). Binding of Quasi-Two-Dimensional Biexcitons. Physical Review Letters, 76(4), 672-675. https://doi.org/10.1103/PhysRevLett.76.672

\section{General rights}

Copyright and moral rights for the publications made accessible in the public portal are retained by the authors and/or other copyright owners and it is a condition of accessing publications that users recognise and abide by the legal requirements associated with these rights.

- Users may download and print one copy of any publication from the public portal for the purpose of private study or research.

- You may not further distribute the material or use it for any profit-making activity or commercial gain

- You may freely distribute the URL identifying the publication in the public portal 


\title{
Binding of Quasi-Two-Dimensional Biexcitons
}

\author{
D. Birkedal, J. Singh,* V. G. Lyssenko, ${ }^{\dagger}$ J. Erland, ${ }^{\ddagger}$ and J. M. Hvam \\ Mikroelektronik Centret, The Technical University of Denmark, DK2800 Lyngby, Denmark
}

(Received 2 October 1995)

\begin{abstract}
Biexciton binding in GaAs quantum wells has been investigated for a range of well thicknesses (80-160 ̊) with spectrally resolved photoluminescence and transient degenerate four-wave mixing. Both light and heavy hole biexcitons are observed. The ratio of the binding energy of the heavy hole biexciton to that of the heavy hole exciton is found to be $\approx 0.2$, and nearly independent of well width over the investigated range. A new theoretical calculation agrees very well with the experimental ratio. This ratio is larger than predicted by Hayne's rule for three-dimensional biexcitons.
\end{abstract}

PACS numbers: 71.35.Cc, 42.50.Md, 73.20.Dx

Since the first reported observation of biexcitons in GaAs/AlGaAs quantum wells [1], a large number of papers concerning different aspects of quantum well biexcitons has been published. Several experimental values of the biexciton binding energy have been reported, but so far no systematic experimental study of the well width dependence of the biexction binding energy has been published. The only published theoretical work on the binding energy of two-dimensional (2D) biexcitons is by Kleinman [2] who predicted a value of $1.2 \mathrm{meV}$ for a $100 \AA$ $\mathrm{GaAs} / \mathrm{AlGaAs}$ quantum well; and indeed the first experimental results seemed to confirm this value $[1,3,4]$. In these experiments, the spectral peak position of the biexciton photoluminescence (PL), or a fit by the line shape, has been used to deduce the biexciton binding energy.

Since then, several authors $[4,5]$ have observed beats in transient four-wave mixing (TFWM), which were ascribed to quantum beats between heavy hole excitons and biexcitons. The use of exciton-biexciton quantum beats is in principle more accurate in determining the biexciton binding energy, since it does not require a fit by a PL line shape. A binding energy of $1.8 \mathrm{meV}$ for a $116 \AA$ quantum well [5] has been determined from TFWM experiments, and in a nonlinear transmission experiment a value of $2.7 \mathrm{meV}$ for a $80 \AA$ quantum well has been found [6]. Recent reports of $1.8 \mathrm{meV}$ for a $100 \AA$ quantum well [7], $1.2 \mathrm{meV}$ for a $250 \AA$ well [8], and $2.8 \mathrm{meV}$ for 50-60 A wells [9] demonstrate that conflicting data have been published by various authors for different samples. Nevertheless, they all indicate higher binding energies for 2D biexcitons than predicted by Kleinman [2]. Recently, Ivanov and Haug [10] have suggested a polariton effect to be responsible for the enhanced binding energy of 2D biexcitons.

Light hole biexcitons have so far not been reported although they should be excited. This is partly due to the fast relaxation of light hole excitons to heavy hole exciton states, which suppresses the light hole exciton luminescence dramatically. In a coherent experiment, the excitonic states are probed before any scattering events take place, making the observation of light hole biexcitons possible. However, the requirement is, as in the case of heavy hole biexcitons, that the biexciton binding energy is larger than, or similar to, the inhomogeneous broadening, meaning that only samples of the best quality will reveal biexcitons.

In this Letter we report (i) the determination of biexciton binding energies in GaAs/AlGaAs quantum wells of different well widths, (ii) the first observation of the light hole biexciton in GaAs quantum wells, and (iii) the result of a theoretical model for calculating the ratio of the binding energy of the biexciton to that of the exciton, using the fractional dimension approach [11]. We determine the binding energies using both coherent and noncoherent optical techniques, i.e., TFWM and PL, respectively. Using spectrally resolved TFWM, we observe light hole biexcitons. They are found to have a lower binding energy than the heavy hole biexcitons and a stronger well width dependence. The ratio of the binding energies of the heavy hole biexciton $\left(E_{b}^{X X}\right)$ to the heavy hole exciton $\left(E_{b}^{X}\right)$ is found to be $\approx 0.2$ independent of well width. Our analytical result for a 2D biexciton gives a value of $E_{b}^{X X} / E_{b}^{X}=0.228$ and independent of the quantum well width, which agree very well with our experimental result. This suggests that a new Hayne's type of rule with a higher constant ratio is valid in dimensions lower than three [12].

To prevent uncertainties from sample to sample fluctuations, one single structure is grown, containing wells of different thicknesses. In this sample, ten wells with thicknesses 80, 100,130, and $160 \AA$ are grown. The wells are separated by $150 \AA \mathrm{Al}_{0.3} \mathrm{Ga}_{0.7}$ As barriers, and after the growth the structure is lifted off the substrate and mounted on a sapphire disk. The samples are kept in a liquid $\mathrm{He}$ cryostat at $5 \mathrm{~K}$ during the experiments.

For the TFWM experiments, the sample was excited by 100 fs pulses from a self-mode-locked Ti:sapphire laser. The laser spot size was focused to approximately $60 \mu \mathrm{m}$ on the sample surface. The TFWM experiments were performed in the two beam self-diffraction geometry [13]. In this geometry, two beams with wave vectors $\boldsymbol{k}_{1}$ and $\boldsymbol{k}_{2}$ are incident on the sample under a small angle. The pulse in the $\boldsymbol{k}_{2}$ direction arrives with a delay of $\boldsymbol{\tau}$ after the pulse in the $\boldsymbol{k}_{1}$ direction. The signal propagates in the $2 \boldsymbol{k}_{2}-\boldsymbol{k}_{1}$ direction. Parallel linearly polarized light in the two input beams has been used in all the experiments. The TFWM 
signal was dispersed in a $0.6 \mathrm{~m}$ spectrometer and detected by an optical multichannel analyzer with a resulting resolution better than $0.1 \mathrm{meV}$. The photoluminescence is excited either by the femtosecond laser or the $632.8 \mathrm{~nm}$ line of a HeNe laser.

The $5 \mathrm{~K}$ absorption spectrum, obtained with a halogen lamp, is shown in Fig. 1(a). We have indicated the heavy hole exciton and light hole exciton absorption lines from the different quantum wells, by $\mathrm{HH}^{X}$ and $\mathrm{LH}^{X}$, respectively. These lines have linewidths from below 1 meV (160 and $130 \AA$ ) to about $1.5 \mathrm{meV}(80 \AA)$. For the PL spectrum in Fig. 1(b), obtained using HeNe laser excitation, we find no Stokes shift for the 160, 130, and $100 \AA$ quantum wells, and a small shift of $0.5 \mathrm{meV}$ for the $80 \AA$ quantum wells. For the 130 and $160 \AA$ quantum wells, a weak light hole exciton luminescence is also observed.

In Fig. 1(b), additional PL peaks are observed below each of the $\mathrm{HH}^{X}$ PL peaks. To investigate whether these PL peaks are due to a transition from the biexciton $(X X)$ to exciton states, we fit the PL data with the line shape used by Phillips et al. [14]:

$$
\begin{aligned}
& I(\hbar \omega) \propto \int g(E ; \hbar \omega) \Theta\left(E_{x}-E_{b}^{X X}-E\right) \\
& \times \exp \left[-\left(E_{x}-E_{b}^{X X}-E\right) / k T_{\mathrm{eff}}\right] d E,
\end{aligned}
$$

where $g(E)$ is a broadening function, $E_{x}$ the exciton energy, $E_{b}^{X X}$ the biexciton binding energy, and $T_{\text {eff }}$ is the

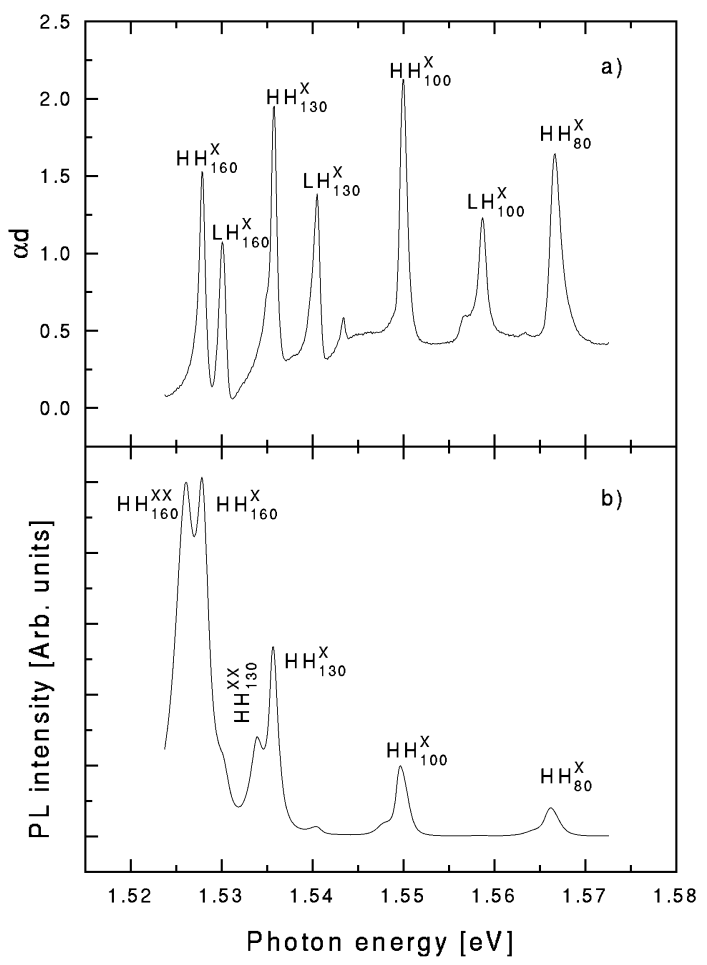

FIG. 1 Low temperature absorption (a) and photoluminescence (b) spectrum. The luminescence data are obtained using HeNe laser excitation. effective temperature of the exciton-biexciton gas. The fits of the biexciton lines suggest binding energies for the 160 and $130 \AA$ quantum wells 1.5 and $1.7 \mathrm{meV}$, respectively. The effective temperature of the biexcitons was determined from the fits to be $12-15 \mathrm{~K}$, which is above the lattice temperature. This temperature increase is due to the HeNe excitation, creating both the excitons and biexcitons with excess energy, and possibly due to "recombination heating" of the exciton gas, as discussed by Phillips et al. [14].

Cold injection of excitons leaves only "recombination heating," as the source for an effective exciton temperature above the lattice temperature. In addition, the cold injection of excitons enhances the creation of biexcitons as suggested by Lovering et al. [4]. To investigate this further, the femtosecond laser was tuned in resonance with the $\mathrm{HH}^{X}$ resonance of the $130 \AA$ quantum well $\left(\mathrm{HH}_{130}^{X}\right)$, and the PL spectra were recorded for different excitation densities. The results of this are presented in Fig. 2(a), showing a superlinear growth of the biexciton luminescence with increasing excitation intensity. The integrated areas under the exciton and biexciton PL lines from the 160 and $130 \AA$ quantum wells are plotted against excitation density in Fig. 2(b) revealing a linear increase in the exciton luminescence and a superlinear growth in the biexciton luminescence as $I_{\mathrm{PL}} \propto I_{L}^{\gamma}$, where $I_{L}$ is the

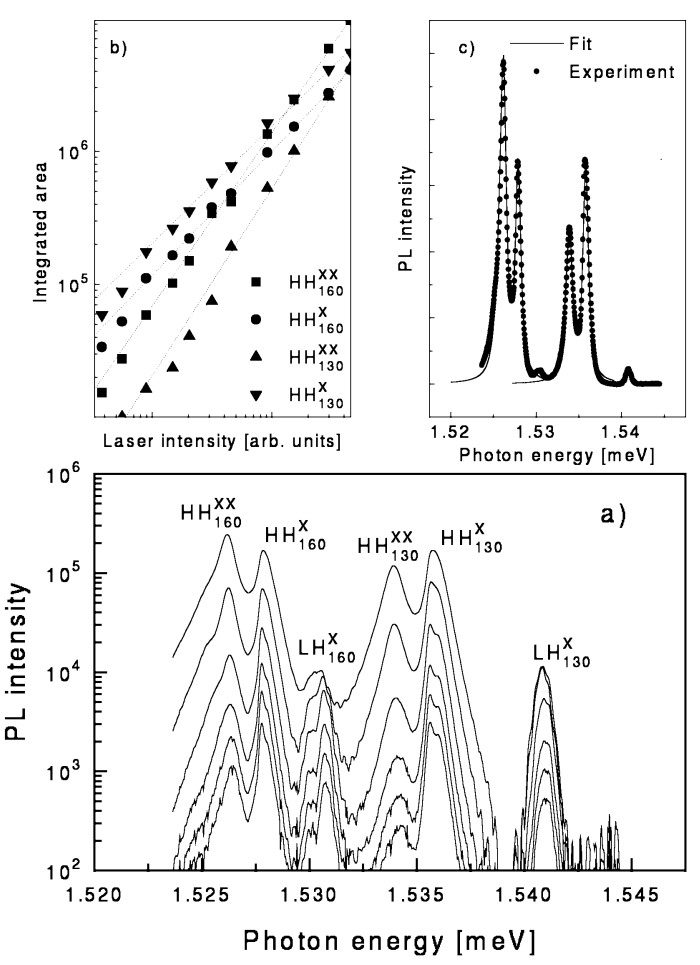

FIG. 2 Resonantly excited PL. (a) The photoluminescence spectra at different excitation densities, (b) linear increase of exciton luminescence and superlinear increase of biexciton luminescence with excitation density, and (c) fit of the photoluminescence lines with the inverted Boltzmann distribution. 
laser intensity and the value of $\gamma$ is determined to be 1.3 for both well thicknesses. The luminescence data have been fitted with Eq. (1), and the result is shown in Fig. 2(c). The PL lines from the heavy hole excitons are fitted by Lorentzians and the step function density of states for the biexcitons have been broadened with Lorentzians of the same width as the exciton line. The effective exciton temperature was in this case 7 $\mathrm{K}$, indicating the pure effect of recombination heating of the initial cold exciton population. These fits gave biexciton binding energies identical to those found using $\mathrm{HeNe}$ excitation.

A weak shoulder below the $\mathrm{LH}_{160}^{X}$ photoluminescence peak, seen in Fig. 2(a), may be related to the light hole biexciton luminescence. However, the density of the light hole excitons and biexcitons is low due to the fast relaxation of light hole excitons to the heavy hole state. In TFWM with 100 fs pulses, we study the exciton states before the initial scattering events take place. By spectrally resolving the TFWM signal, the contributions to the signal from individual states can be identified. With the laser resonant to the $\mathrm{HH}_{130}^{X}$, the TFWM spectrum is shown in Fig. 3 for a delay of 3 ps. Distinct lines are observed for all the exciton resonances. Below the exciton resonances clear lines are seen. These lines coincide with the lines observed in the luminescence and are identified as being due to two-photon transitions to the biexciton states via the intermediate exciton states. Since the spectral width of the laser is much wider than the biexciton binding energy, photons with an energy equal to the exciton energy minus the biexciton binding energy are emitted for both positive and negative delays. Note that the light hole biexciton in the $130 \AA$ quantum well is clearly resolved, with a binding energy of $1.4 \mathrm{meV}$. Using cocircular polarized light in the input beams, the contribution from the biexciton reso-

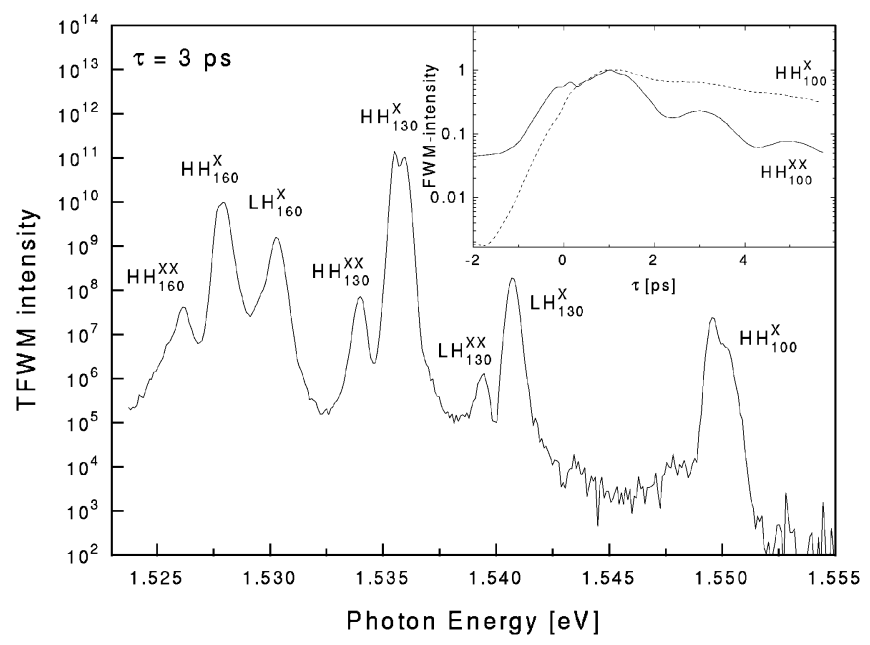

FIG. 3 Spectrally resolved four-wave mixing at $\tau=3$ ps showing the heavy hole and light hole biexcitons. Inset shows the four-wave mixing instensity of the heavy hole exciton and biexciton as a function of delay.

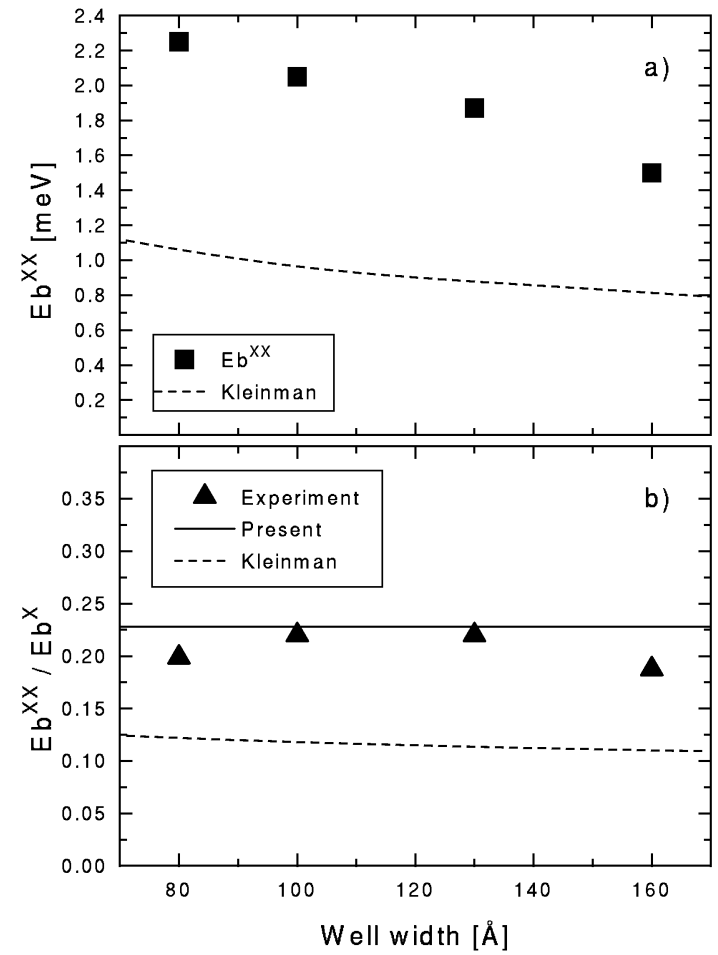

FIG. 4 Well width dependence of binding energies. (a) Heavy hole binding energies, and (b) ratio of the heavy hole biexciton to exciton binding energy. The dashed curves in (a) and (b) indicate the calculation of Kleinmann [2], the full curve in (b) shows the result of the present calculation, and the markers indicate the experimental data.

nances to the TFWM spectrum disappears, confirming the assignment [15]. The inset of Fig. 3 shows the intensity of the TFWM signal at the $\mathrm{HH}_{100}^{X}$ and $\mathrm{HH}_{100}^{X X}$ spectral positions, with the laser peak intensity at $\mathrm{HH}_{100}^{X}$. A clear beat is seen in the biexciton component with a beat period of $2.00 \mathrm{ps}$ corresponding to $2.07 \mathrm{meV}$ in good agreement with the $2.05 \mathrm{meV}$ splitting between the exciton and biexciton lines extracted from the TFWM spectra. By appropriate tuning of the laser, we obtain the response from the $\mathrm{HH}^{X X}$ in each of the quantum wells, and determine their binding energies. The well width dependence of the $\mathrm{HH}^{X X}$ binding energies is shown in Fig. 4(a). Also shown, as a dashed curve, is the calculation by Kleinman [2].

Assuming a square structure for a 2D biexciton, with two electrons and two holes separated by diagonals $2 r$, we have calculated the ratio of the binding energy of biexcitons to that of excitons analytically using the fractional dimension approach [11]. Our model structure helps in reducing the biexciton Hamiltonian into an exciton Hamiltonian with a modified reduced mass and modified dielectric constant, which in spherical coordinates reads:

$$
\begin{aligned}
\hat{H}_{X X}= & -\frac{\hbar^{2}}{2 \mu_{X X}}\left[\frac{1}{r^{\alpha-1}} \frac{\partial}{\partial r}\left\{r^{\alpha-1} \frac{\partial}{\partial r}\right\}\right] \\
& +\frac{L^{2}}{2 \mu_{X X} r^{2}}-\frac{e^{2}}{\epsilon_{X X} r},
\end{aligned}
$$


where $\mu_{X X}=\frac{2}{3} \mu_{X}$, and $\epsilon_{X X}=\sqrt{2} \epsilon_{X} /(4-\sqrt{2}) . \quad \mu_{X}$ and $\epsilon_{X}$ are the reduced effective mass and dielectric constant of the material, respectively. It is to be noted that for the present case the fractional dimension parameter is $1 \leq \alpha \leq 2$, to conform with our proposed geometrical model of a biexciton confined in a quantum well. $L^{2}$ is the square of the angular momentum operator of a 3D biexciton. The biexciton binding energy is thus derived as a function of the exciton binding energy:

$$
E_{b}^{X X}=\left[\frac{\mu_{X X}}{\mu_{X}} \frac{\epsilon_{X}^{2}}{\epsilon_{X X}^{2}}-2\right] E_{b}^{X},
$$

which gives $E_{b}^{X X} / E_{b}^{X} \simeq 0.228$, independently of the fractional dimension $\alpha$ and hence the well width. The details of this calculation will be published elsewhere [16].

The binding energies of the heavy hole excitons have been estimated from photoluminescence excitation measurements, using a halogen lamp and a monochromator. In Fig. 4(b), we have compared the experimental ratio of the heavy hole biexciton binding energy and the heavy hole exciton binding energy $E_{b}^{X X} / E_{b}^{X}$ with those of Kleinmann [2] and the present calculation. The experimental results deviate significantly from the calculation by Kleinman but agree quite well with our calculation, and suggest that a Hayne's type of rule, with a higher value of the ratio, is found in lower dimensional systems. Our experimental findings indicate that the binding energy of the light hole biexciton is lower than that of the heavy hole biexcitons. Our theoretical result (3) gives a ratio that is independent of the mass. However, due to the larger experimental uncertainties involved in determining the binding energies of both of the light hole excitons and the light hole biexcitons it is not possible at the present to address this issue any further.

In summary, this is the first report of light hole biexcitons in GaAs quantum wells. The quantum well biexciton binding energies have been measured and calculated for a series of different well thicknesses. The ratio of the biexciton and exciton binding energies is found to be independent of well width having a value of $\approx 0.2$ in agreement with the results of a theoretical model. We find larger values of the binding energies than those initially found for $100 \AA$ A quantum wells $[1,3,4,14]$. However, our results agree with those published in a number of recent reports [5-9], on larger biexciton binding energies in high quality samples. Since the present theoretical approach is general with respect to the quantum well host material, we believe that our findings are of importance also in the discussion of the biexcitonic origin of the lasing mechanism in wide gap II-VI semiconductors [17].

We would like to thank C.B. Sørensen, III-V NANOLAB, for the sample preparation. This work was supported by the Center Nanostructures (CNAST) and the Danish Natural Science Research Council.

*Permanent address: Faculty of Science, Northern Territory University, Darwin, NT 0909, Australia.

†Permanent address: Institute of Microelectronics Technology, and Superpure Materials, Chernogolovka, Moscow District 142432, Russia.

*Present address: Institute of Physics and Astronomy, University of Aarhus, 8000 Aarhus C, Denmark

[1] R. Miller, D. Kleinman, A. Gossard, and O. Monteanu, Phys. Rev. B 25, 6545 (1982).

[2] D. Kleinman, Phys. Rev. B 28, 871 (1983).

[3] R. Cingolani, Y. Chen, and K. Ploog, Phys. Rev. B 38, 13478 (1988).

[4] D. Lovering, R. Phillips, G. Denton, and G. Smith, Phys. Rev. Lett. 68, 1880 (1992).

[5] K.-H. Pantke et al., Phys. Rev. B 47, 2413 (1993).

[6] S. Bar-Ad and I. Bar-Joseph, Phys. Rev. Lett. 68, 349 (1992).

[7] J. Kim, D. Wake, and J. Wolfe, Phys. Rev. B 50, 15099 (1994).

[8] G. Smith, E. Mayer, J. Kuhl, and K. Ploog, Solid State Commun. 92, 325 (1994); E. J. Mayer et al., Phys. Rev. B 50, 14730 (1994).

[9] B. Deveaud (private communication).

[10] A. Ivanov and H. Haug, Phys. Rev. Lett. 74, 438 (1995).

[11] X. F. He, Phys. Rev. B 43, 2063 (1991).

[12] J. R. Haynes, Phys. Rev. Lett. 4, 361 (1960).

[13] T. Yajima and Y. Taira, J. Phys. Soc. Jpn. 47, 1620 (1979).

[14] R. Phillips, D. Lovering, G. Denton, and G. Smith, Phys. Rev. B 45, 4308 (1992).

[15] H. H. Yaffe, Y. Prior, J. P. Harbison, and L. T. Florentz, J. Opt. Soc. Am. B 10, 578 (1993).

[16] J. Singh, D. Birkedal, V. G. Lyssenko, and J. M. Hvam (unpublished).

[17] F. Kreller, M. Lowisch, J. Puls, and F. Henneberger, Phys. Rev. Lett. 75, 2420 (1995). 